\title{
Halo of a High-Brightness Electron Beam
}

\author{
G. Haouat, N. Pichoff, C. Couillaud, J.P. De Brion, J. Di Crescenzo, S. Joly, A. Loulergue, \\ C. Ruiz, S. Seguin, and S. Striby, CEA-BIII/SPTN, BP 12, 91680 Bruyères-le-Châtel. France.
}

\begin{abstract}
The experimental study of transverse profile of the ELSA-linac electron beam has been undertaken in order to analyze the mechanisms of halo formation and development in the generation, acceleration and transport of a highintensity, high-brightness charged-particle beam. Measurements are reported in which the beam profile is observed over four to five decades. They were performed using an imaging technique in which light emitted from an optical transition radiation (OTR) screen placed in the beam path is transported through conventional optics to an intensified video camera. The electron-density distribution is shown to be dependent on the beam current, through space charge effects, initial conditions and transport configuration.
\end{abstract}

\section{INTRODUCTION}

Technology of high-intensity linear accelerators has progressed so rapidly in the past decade that they are now being considered for use in a number of applications. Possible applications are isotopic separation or plasma heating with high-power free electron lasers fed by electron linacs, or nuclear waste management, production of tritium, transmutation of defense and commercial plutonium stocks with intense spallation-neutron sources driven by large proton or light-ion linear accelerators. However, experimental observations and multiparticlesimulations indicate that highintensity beams in accelerators develop a low-density halo at the periphery of the central core of the beam. This outer part of the distribution leads to particle losses in the accelerator which, for a high-energy beam, may induce enough activity or radiation damage in the structures to complicate considerably maintenance and operation of the machine. The major challenge is to control the beam profile, which implies a better knowledge of the phenomena involved in halo formation.

Several theoretical studies and numerical-simulation works have addressed the problem of beam halo formation and development in high-intensity linear accelerators. However, predictions of the simulations may be quantitatively incorrect since, because of computer capabilities, halo properties are described by only a few particles. Therefore, experimental study of beam halo is necessary to check theoretical predictions and complement simulations.

Few experiments, to date, have been dedicated to precise determination of the beam structure extending in the very low density regions constituting the halo. We have undertaken, at Bruyères-le-Châtel, an experimental study of the transverse spatial distribution of the high-intensity, high-brightness electron beam delivered by the ELSA linac. The objective of this study is to analyze the beam profile over a very large dynamic range under varying conditions in the generation, acceleration and transport of the beam. An experiment is described in which the beam profile has been observed over at least four decades.

\section{EXPERIMENTAL TECHNIQUE}

The ELSA facility has been described previously [1]; only features peculiar to our halo experiment are given here. The accelerator, which consists in a photoinjector cavity followed by three accelerating cavities, delivers a highbrightness space-charge dominated electron beam. The 2$\mathrm{MeV}$ photoinjector cavity and its anode coil are shown schematically in Figure 1. The focusing anode coil is of particular importance since it counteracts the space-charge defocusing effect on the beam, which is important at low electron energy.

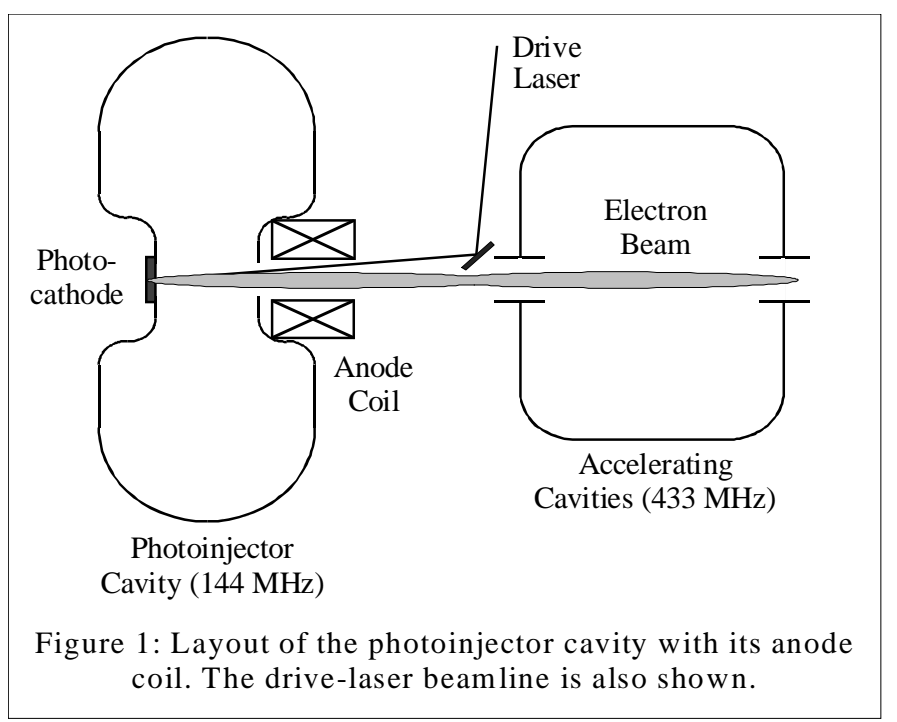

The experimental apparatus designed for the halo measurements was installed at the end of the linac. It is a modified version of the devices used to diagnose transverse profile on the ELSA facility [2]. The setup, which has been described in detail in a previous paper [3], consists of an electron/photon conversion screen, a transport optics and a video camera. The converter, which is moved in the beam path by an actuator, is an optical transition radiation (OTR) screen. This type of screen is suitable for accurate halo measurements since it has an excellent linear response over a 
wide dynamic range, good spatial resolution, a reasonable sensitivity, and does not suffer saturation effects or image smearing induced by thermal effects. The screen plane is oriented at $45^{\circ}$ with respect to the beam axis so that the backward lobe of radiation is observed at $90^{\circ}$ to the beam through a quartz window and conventional image-transport optics to a variable-gain video camera coupled to a dataacquisition system. The optical system has been designed for an observation field of $16 \mathrm{~mm}$ at the screen, corresponding to roughly 10 times the core diameter of a beam of $20 \pi$ mm.mrad normalized emittance transported under optimum conditions.

\section{THE HOLE-BORED SCREEN METHOD}

The hole-bored screen method was used for the halo measurements. The experimental procedure consists in moving an OTR screen into the beam path. The screen has the form of a stainless-steel disk, 1-mm thick and 50-mm in diameter, with a hole bored in the center. The disk is polished to optical standards. The electron-beam core is first observed with its image below saturation, by appropriate setting of the light intensifier. In this measurement, the beam is slightly offcenter with respect to the hole in the disk. The beam is then steered toward the hole through which most of the core electrons pass. Then, the halo can be properly observed by increasing amplification of the light intensifier. This method yields better results than the saturated-core method, described in reference [3], since undesirable phenomena, which can spoil observation of the halo, are eliminated and background light is considerably reduced.

The measurements were performed with three OTR screens having hole diameters of 2.5, 4.0 and $6.0 \mathrm{~mm}$. They were changed, during the experiment, to fit the size of the beam core to be observed. These three screens together with a light intensifier with 7-decade dynamic range permitted the observation of density profiles over five orders of magnitude. The electron beam, of $17.5 \mathrm{MeV}$ energy, consisted of
macropulses of $100-\mu$ s duration, at a repetition rate of $1 \mathrm{~Hz}$. The macropulse train was composed of $\sim 20$-ps long bunches spaced $69.2 \mathrm{~ns}$ apart; the bunch temporal profile was gaussian. Bunch charges of 0.5 to $3 \mathrm{nC}$, corresponding to peak currents of 25 to $150 \mathrm{~A}$, were obtained by adjusting the drive-laser beam intensity. The laser spot at the photocathode was circular with a diameter of $4 \mathrm{~mm}$, and illumination was almost uniform over the spot surface.

\section{DATA ACQUISITION AND PROCESSING}

For each setting of the accelerator parameters, images were taken with two to three different combinations of OTR screens and light intensifier gains in order to cover the largest electron-density range. Background images were also taken by switching off the drive-laser light. In off-line data processing the profile images were normalized one to the other, by using the precise calibration of the light intensifier, to finally yield the electron-beam transverse distribution after background has been subtracted.

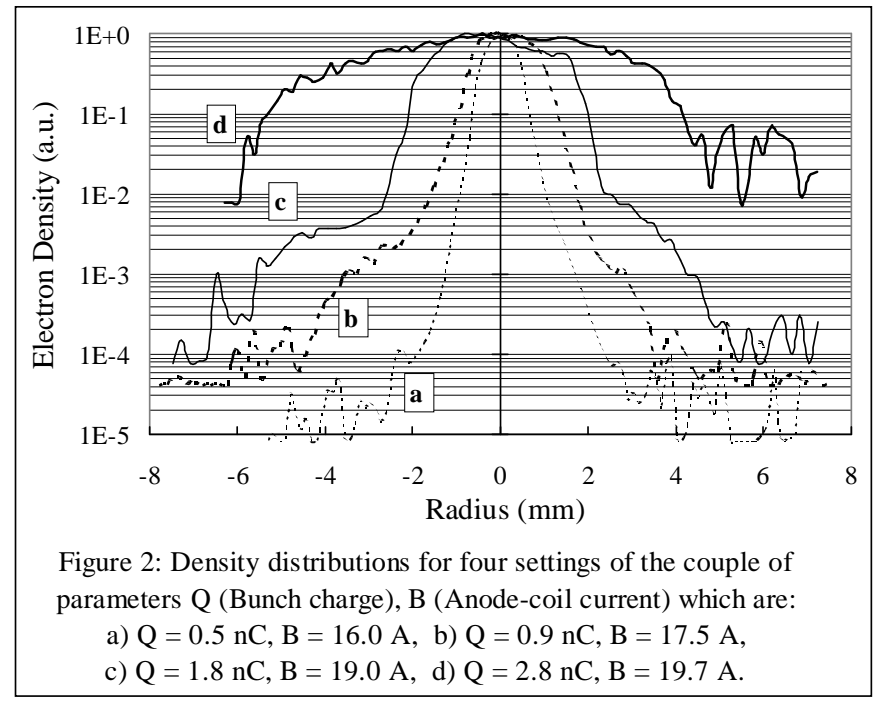

Once the transverse profile has been reconstructed horizontal or vertical cross sections of the electron-density distribution can be deduced. Beam profiles, which were measured for different values of the two parameters, bunch charge and anode-coil current, are presented in Figure 2 and will be discussed below. This figure shows that our experimental setup is capable of measuring density distributions over 4 to 5 decades.

\section{RESULTS AND INTERPRETATION}

In the present work we have investigated the influence on the beam transverse profile of the electron-bunch charge and of the current in the focusing anode coil of the ELSA photoinjector.

\section{A. Results}

The transverse profile has been observed for bunch charges (Q) ranging from 0.5 to $3.0 \mathrm{nC}$ and anode-coil current (B) varying between 15.0 and $19.7 \mathrm{~A}$. Data were taken in four separate runs for 20 sets of Q,B values. Horizontal cross sections of the transverse density distribution have been extracted from the reconstructed profile images. Characteristic spectra are displayed in Figure 2 , showing important changes in the beam density profile.

Observation of the data leads to two remarks:

- Beam size changes strongly. At low anode-coil currents $\left(\begin{array}{ll}\sim & \mathrm{A}\end{array}\right)$, there is a linear beam-spot broadening with increasing bunch charge, while, at higher anode-coil currents ( $>18 \mathrm{~A}$ ), the spot size is large and almost constant with charge. The low-coil-current behaviour has been observed before and attributed to space-charge effects [3].

- Profile shapes look quite different with the anode-coil current. i) At low currents $(<16 \mathrm{~A})$, the density profile 
decreases almost exponentially from the center of the beam towards outside. $i i)$ At mid currents $(\sim 18$ A), the density profile seems to be composed of two gaussian shapes, as if two beams, with different transport properties, were travelling together. The more dense beam could be called the "core", the other one, the "halo". Curves b) and c) of Figure 2 exhibit this bi-gaussian shape. iii) At high currents (>19.5 A), the core and the halo seem to be completely mixed.

\section{B. Interpretation}

The density profiles have been processed to yield an estimate of the fraction of the beam that extends beyond a given distance from the centroïd of the beam. The resulting data have been used to deduce, for each measurement, the beam radii enclosing $90 \%, 99 \%, 99.9 \%$ and $99.99 \%$ of the particles. The 20 measurement results have then been analyzed using an interpolation-extrapolation technique to yield the radii variations in the Q,B space. Variations of the beam radius enclosing $99 \%$ of the particles are displayed in Figure 3 as a 3D contour plot; the data are labelled as "EXPERIMENT".

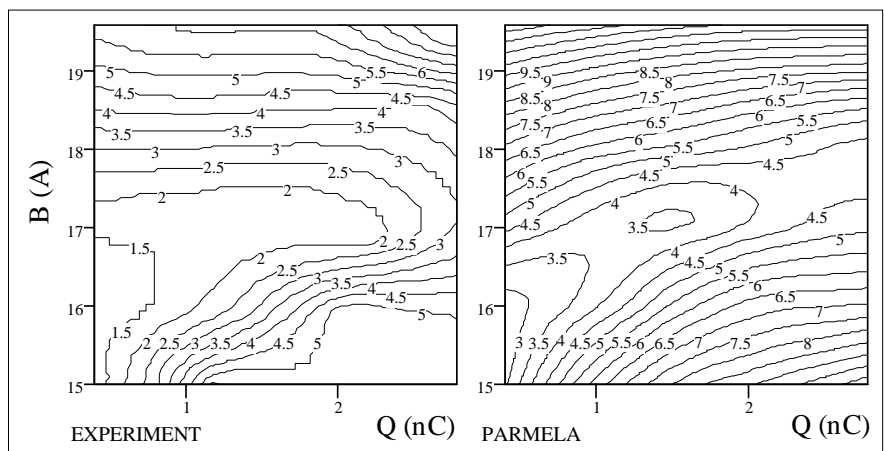

Figure 3: Variations of the $99 \%$ beam radius with bunch charge $\mathrm{Q}$ and anode-coil current $\mathrm{B}$.

The experiment has been simulated with the code PARMELA, in order to explain our results and to try to understand the physical phenomena involved in the halo formation and development. Because of the limited numerical capabilities of the code contour plots could not be obtained for beam radii enclosing more than $99 \%$ of the particles. The data for a beam radius enclosing $99 \%$ of the particles are presented in Figure 3; they are labelled as "PARMELA". Comparison of contour plots for measured data and simulation results shows good qualitative agreement, but the simulation yields radii systematically larger than the experiment by a factor of roughly 2 . This discrepancy may be explained by a crude simulation of the photoinjector or an unrealistic estimate of the transverse focusing strength of the accelerating cavities.

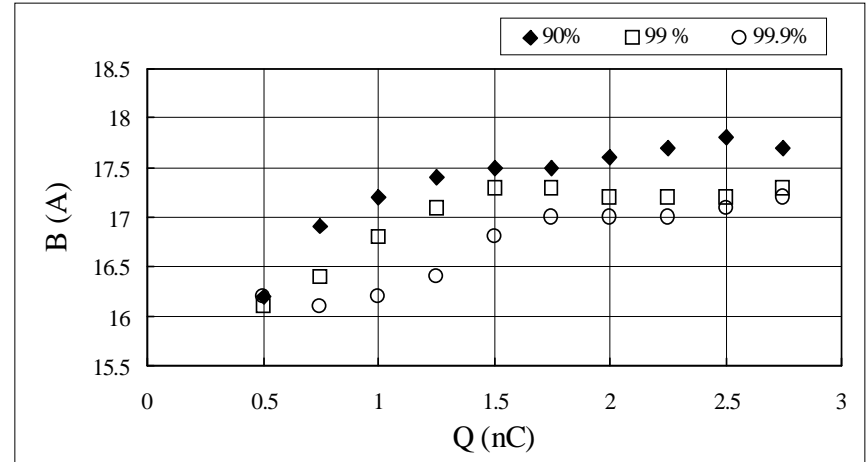

Figure 4: Experimental curves $\mathrm{B}=\mathrm{f}(\mathrm{Q})$ corresponding to minimum radii enclosing $90 \%, 99 \%, 99.9 \%$ of the particles.

Both plots exhibit a valley at the same location in the Q,B space. The bottom of this valley represents the optimum anode-coil current at a given bunch charge for transporting an electron beam of minimum size. The curves corresponding to the lowest path in the valley have been estimated for both experimental and simulation results, and they compare satisfactorily. The experimental $\mathrm{B}=\mathrm{f}(\mathrm{Q})$ curves corresponding to minimum beam-radii enclosing $90 \%, 99 \%$ and $99.9 \%$ of the particles are displayed in Figure 4. It indicates that the anode current should be higher if the objective is to optimize the transport of the beam core only, and relatively lower if the objective is to optimize the transport of the core+halo.

\section{CONCLUSION}

The hole-bored OTR screen and the imaging technique constitute powerfull tools for measuring electron-beam transverse distributions over four to five orders of magnitude and for studying the halo in the transport of a high-brightness beam. The analysis of the influence on the beam profile of the bunch charge and the focusing strength of the ELSA photoinjector yields interesting results. Further work is planned to investigate the role of other parameters controlling the beam, and to use more extensively the simulation tools in order to understand the physics of the halo.

\section{REFERENCES}

[1] R. Dei-Cas et al., "Status report on the lowfrequency photo-injector and on the infrared FEL experiment ELSA", Nucl. Inst. Meth. A296 209 (1990).

[2] G. Haouat, C. Couillaud, J. Di Crescenzo, J. Raimbourg and S. Seguin., Proc. First European Workshop on Beam Diag. and Inst. for Part. Acc., Montreux, Switzerland. CERN PS/93-35 (BD) - CERN SL/93-35 (BI) (1993) 180.

[3] G. Haouat, N. Pichoff, C. Couillaud, J. Di Crescenzo, S. Joly, S. Seguin and S. Striby, "Experimental study of the ELSA electron-beam halo", BIW94, Beam Instrumentation Workshop - Vancouver, B.C., Canada, 2 - 6 Oct. 1994. 